\title{
NUEVOS REGISTROS DE DISTRIBUCION Y NOTAS BIOLOGICAS DE CYDIA LARGO HEPPNER (LEPIDOPTERA: TORTRICIDAE)
}

\section{NEW DISTRIBUTIONAL RECORDS AND BIOLOGICAL NOTES OF CYDIA LARGO HEPPNER (LEPIDOPTERA: TORTRICIDAE)}

\author{
Héctor A. Vargas ${ }^{1} \&$ Luis E. Parra ${ }^{2}$ \\ ${ }^{1}$ Departamento de Recursos Ambientales, Facultad de Ciencias Agronómicas, Universidad de Tarapacá, Casilla 6-D, \\ Arica, Chile..Email: havargas@uta.cl \\ ${ }^{2}$ Departamento de Zoología, Facultad de Ciencias Naturales y Oceanográficas, Universidad de Concepción, Casilla \\ 160-C, Concepción, Chile.
}

\begin{abstract}
Two new distributional records for Cydia largo Heppner, in Azapa and Chaca valleys, northern Chile, are reported. Some observations about of the biology of $C$. largo are presented.
\end{abstract}

Azapa y Chaca son dos valles costeros ubicados en el extremo norte de Chile. Para algunos grupos taxonómicos, estos valles presentan gran afinidad con los valles costeros del sur de Perú, y en conjunto poseen varias especies vegetales y animales endémicas, lo cual permite definirla como la subprovincia biótica "Desértica Costera" dentro del Neotrópico (Porter 1985). Al contrario, estas localidades pueden indicar el límite sur para taxa ampliamente distribuidos a través del continente americano (Angulo \& Jana-Sáenz 1983, Artigas \& Angulo 1980).

Mediante prospecciones efectuadas en Azapa y Chaca, como parte de un estudio de lepidópteros asociados a yaro, Acacia macracantha Willd. (Mimmosaceae), se detectó en ambos valles la presencia de Cydia largo Heppner (Fig. 1), cuya distribución geográfica previamente conocida estaba restringida a Florida (Estados Unidos) y Cuba (Heppner 1981). Por lo tanto, el objetivo de esta nota es reportar nuevos registros que amplían notablemente la distribución conocida para C. largo. Algunas observaciones sobre la biología de la especie son también indicadas.

El material examinado fue colectado al estado larvario sobre inflorescencias de yaro entre los meses de noviembre y diciembre de 2002 en los valles de Azapa y Chaca. Las larvas fueron mantenidas en frascos de vidrio y alimentadas con inflorescencias de yaro. Periódicamente los frascos fueron observados para verificar la emergencia de adultos, los cuales fueron acondicionados para ser incorporados a la Colección Entomológica de la Universidad de Tarapacá (IDEA). La identificación fue efectuada sobre la base de la literatura disponible (Heppner 1981). Posteriormente fue confirmada por el Dr. Richard L. Brown, Mississippi State University, USA.

$$
\text { Cydia largo Heppner, } 1981
$$

Material examinado. CHILE. ARICA. Adultos

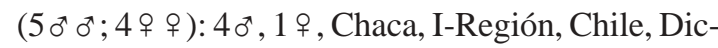
2002, Flor Yaro. H.A. Vargas coll. $10^{\star}, 2$ 우, Chaca, I-Región, Chile, Dic-2002, Flor Yaro, Y. Cortés, coll. 1 ㅇ, Azapa, I-Región, Chile, Nov-2002, Flor Yaro, H.A. Vargas, coll. (IDEA).

Distribución. Se detectó la presencia de $C$. largo en las localidades de Azapa (18 ${ }^{\circ} 34^{\prime} \mathrm{S}$; 70 00’ O) y Chaca ( $\left.18^{\circ} 48^{\prime} \mathrm{S} ; 70^{\circ} 07^{\prime} \mathrm{O}\right)$. Estas localidades representan dos nuevas áreas de distribución para esta especie.

OBSERVACIONES BIOLÓGICAS. Acacia macracantha es mencionada por primera vez como planta hospede- 
ra de larvas de $C$. largo, las cuales tienen hábitos antofágicos. En las inflorescencias de yaro las larvas consumen diversas partes florales e internamente el receptáculo. Una vez completada su alimentación, las larvas de último instar descienden y se ubican en el interior de hendiduras del tronco o en la hojarazca, donde construyen un capullo de seda y posteriormente pupan. Se desconoce el número de generaciones anuales que C. largo completa en el área de estudio. Sin embargo, colectas posteriores han permitido constatar que se trata de una especie multivoltina. La distribución previamente conocida para C. lar- go incluía sólo a la localidad tipo Florida (Estados Unidos) y Cuba (Heppner 1981). Los nuevos registros indicados en el presente trabajo (Azapa y Chaca) extienden la distribución conocida para esta especie, y permiten suponer que probablemente se encuentre ampliamente distribuida a través del continente americano. Sin embargo, futuras prospecciones permitirán verificar la real distribución geográfica de C. largo y descartar la posibilidad de que sea una especie de distribución disyunta, tal como queda documentado hasta ahora, considerando los antecedentes indicados en la presente nota.

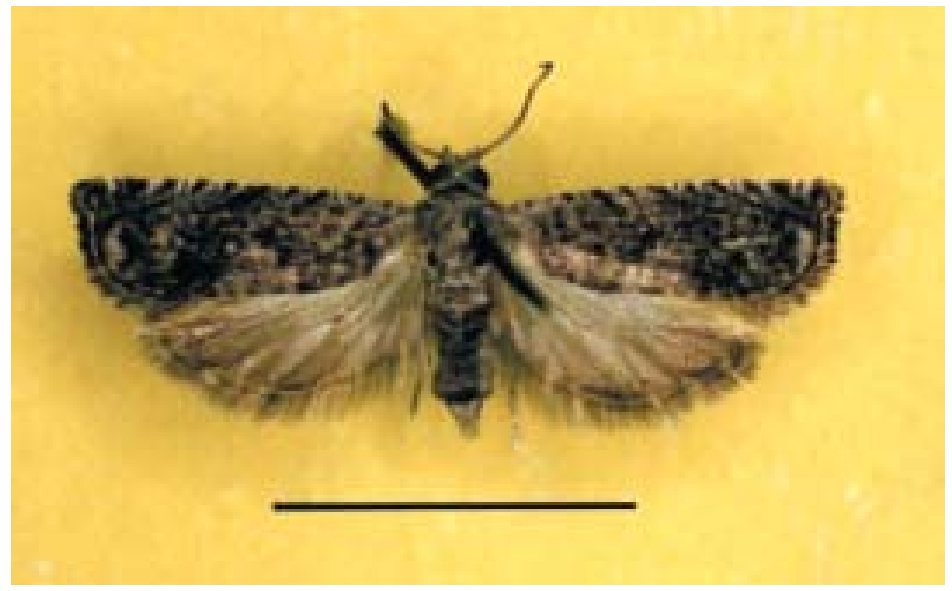

Figura 1. Cydia largo Heppner. Adulto $\circ$ en vista dorsal. Escala: 0,5 cm.

Figure 1. Cydia largo Heppner. Adult 9 in dorsal view. Scale: $0,5 \mathrm{~cm}$.

\section{AGRADECIMIENTOS}

Al Dr. Richard L. Brown, Mississippi State University, USA, por confirmar la identificación y a la Dirección de Investigación, Universidad de Tarapacá, Proyecto DIPOG 9704-03, por el apoyo financiero.

\section{BIBLIOGRAFIA}

Angulo, A. O. \& C. JAnA-SÁEnz. 1983. Catálogo crítico, ilustrado y claves de Catocalinae y Ophiderinae para Chile (Lepidoptera: Noctuidae). Gayana (Zoología) 45: 3-26.

Artigas, J. N. \& A. O. Angulo. 1980. Revisión del género Mallophora Macquart por sistemática alfa y taxonomía numérica (Diptera: Asilidae). Gayana (Zoología) 43: 5-182.

Heppner, J. B. 1981. A new Cydia (Lepidoptera: Tortricidae) from Florida and Cuba. Journal of the Lepidopterists' Society 35(4) 278-280.

Porter, C. C. 1985. Trachysphyrus and the new genus Aeliopotes in the Coastal Desert of Peru and north Chile (Hymenoptera: Ichneumonidae). Psyche 92(4) 513-545.

Fecha de recepción: 10.09.05

Fecha de aceptación: 03.07.06 